\title{
PREDICTING THE ACCEPTANCE OF DENTAL CARE BY RESIDENTS OF NURSING HOMES
}

\author{
By S. Stephen Kegeles, Ph.D." \\ Stanley Lotzkar, D.D.S., M.P.H. * \\ and Lewis W. Andrews, M.P.H.*
}

\begin{abstract}
Inasmuch as prediction should be simpler and cheaper than planning and completing a survey, you are advised to read this paper.
\end{abstract}

It has become evident that the utility of basic health and scientific findings is limited by the unwillingness of certain individuals to take the actions necessary to prevent or ameliorate disease. This failure to participate in programs of public health has been of great concern to public health personnel always, but has achieved increasing importance in recent years.

The population least likely to participate in the case-finding programs of public health and in the programs for which it was necessary to pay for services has been the population over age $65 .^{1,2,3}$ The need for health of this population that lives in nursing homes or is chronically ill at home only now has begun to be known to personnel in public health.

Information is needed about (1) the extent of the health-needs of this population and (2) the likelihood of this population accepting services directed toward fulfilling these needs. This need for information now has been enhanced by the advent of the new provisions of Medicare.

The Division of Dental Public Health of the U. S. Public Health Service, in connection with the Community Studies, Inc., which represented the metropolitan community of Kansas City and the Kansas City School of Dentistry, organized a project of research and demonstration in Kansas City, Missouri, during the period of June 1957 to June 1961, to ascertain the dental needs of persons in nursing homes and the home-bound, chronically ill persons in order to assess the extent of dental services required to meet these dental needs. A publication concerned with the findings from the demonstration since has been issued. ${ }^{4}$ As part of this larger study of a community, a limited social survey was formulated and conducted: (I) to identify the characteristics of individuals most likely to accept dental services, and (2) to provide a basis of formulating hypotheses useful in understanding why these groups accepted or failed to accept free dental treatment.

\footnotetext{
*Associate Professor of Public Health Administration, School of Public Health, The University of Michigan, Ann Arbor.

* Assistant Chief, Manpower and Education Branch, Division of Dental Health, U. S. Public Health Service, Bethesda, Maryland.

* * Senior Public Health Advisor, U. S. Public Health Service, Kansas City, Missouri.

*A brief statement concerning the social survey was presented in the larger document concerned with the Kansas City Study $^{4}$ (pages 25, 26).
} 
The primary objective of this social survey was to develop a very simple set of questions to predict which individuals were likely to accept free dental treatment.

\section{Method}

The interviewing form used for this study consisted of 14 questions, each of which required one-word answers. It included (1) a section concerned with present and past dental behavior and individual desires for dental care, (2) indirect nondental items, (3) items of particular relevance to the psychology of older persons, and (4) a question about intention to take advantage of service if offered. The questions follow as Table 1 .

TABLE 1

\section{QUESTIONS USED FOR SOCIAL SURVEY}

1. Before you came here, how often did you have your teeth cared for by a dentist?

Twice a year

Once a year

Every two years

Less often than every two years

Never

2. Since you have been here, have you seen a dentist?

3. How recently was that?

Within the last year

Within the last two years

Longer ago than two years

4. Since you have been here, have you felt you needed to see a dentist?

5. Would you get dental care now if you needed it?

6. Do you have dental plates?

7. I see you are not wearing your dental plates. is that because:

They are broken

They don't fit

You forgot to put them in

You don't want or need them

They are lost

8. How much does it bother you to have a dentist work on your teeth? Would you say it bothers you:

Very much

Some

Little

None at all

9. Do you ever get yourself all dressed up for any occasion?

10a. For men: How often would you say you shaved?

10b. For women: How often would you say you fixed your hair?

At least once a day

About every other day

Less frequently than every other day 
11. How often would you say you brushed your teeth (or "cleaned your dentures")?

At least once a day

About every other day

Less frequently than every other day

12. Are you interested in making new friends?

13. Do you have difficulty in finding things to do to keep busy?

14. If you were offered dental treatment at the dental clinic, would you be:

Willing to accept such treatment?

Unwilling to accept such treatment?

Two experienced interviewers were trained over a three-hour period to administer the interview. They then interviewed all patients (about 80 percent of the total population examined), disclosed in a prior dental examination as needing treatment and judged capable of receiving such treatment, in each of the first 29 nursing homes selected for assessment by the project's staff. This group amounted to a total of 310 patients that was exposed to an interview of about 10 minutes in duration. Later, a social worker discussed and interpreted the available services to each of the patients as an inducement for acceptance of treatment. Treatment was available in the dental clinic of the study or through private dental facilities. Transportation was made available to bring patients to and from the clinic where treatment was provided. An independent analysis was made of the objective dental needs of these patients by the second participant in this paper as a part of the total project prior to any interviewing.

The data collected included (1) demographic factors (age, sex, race, type and size of nursing home), (2) the answers to the interviewer's questions, (3) a measurement of objective dental needs, and (4) a record of the actual acceptance or rejection of treatment by each of the patients.

Data were screened rapidly by the use of computed tables of significance for fourfold tests of contingency." Chi-squares then were computed on the findings which were found to be significant* by this rapid-screening process.

\section{Findings}

The findings to be presented are related to (1) demographic factors, (2) responses to interview, and (3) objective dental needs on the basis of acceptance or refusal of dental services.

\section{Relationship Between Demographic Factors and Acceptance of Treatment}

Seventy percent of the group accepted the available treatment. Persons under 75 years of age accepted treatment more frequently than persons over 75 years of age (Table 2). Negroes accepted treatment more frequently than did whites. Only three of 27 Negroes refused treatment (Table 2).

\footnotetext{
"Reports will be submitted of significant findings only. Significance has been defined to mean that the finding could not have occurred by chance in more than five instances out of 100 . Many of the findings could not have occurred by chance in more than one instance out of 100 and some findings could not have occurred by chance in more than one instance out of 1000 .
} 
TABLE 2

RESPONDENTS ACCEPTING TREATMENT BY AGE, SEX, AND RACE

\begin{tabular}{|c|c|c|c|c|}
\hline & Number & Accepting & Treatment & \\
\hline & Respondents & Number & Percent & \\
\hline TOTAL & 310 & 215 & 69.4 & \\
\hline Age * & & & & \\
\hline Under 50 & 13 & 13 & 100.0 & \\
\hline $50-64$ & 34 & 26 & 76.5 & \\
\hline $65-74$ & 85 & 61 & 71.8 & \\
\hline Total under 75 & 132 & 100 & 75.8 & $X^{2}=4.15$ \\
\hline Total $75 \&$ over & 177 & 115 & 65.0 & $p=.05$ \\
\hline Sex & & & & \\
\hline Male & 115 & 93 & 80.9 & $X^{2}=11.38$ \\
\hline Female & 195 & 122 & 62.6 & $p=.001$ \\
\hline Race & & & & \\
\hline White & 283 & 191 & 67.5 & $X^{2}=5.16$ \\
\hline Negro & 27 & 24 & 88.9 & $p=.05$ \\
\hline
\end{tabular}

*The age of one individual was not determined.

\section{Relationship Between Responses to the \\ Questionnaire and Acceptance of Treatment*}

Individuals who stated that they had felt a need to see a dentist during their stay in the nursing home accepted treatment more frequently than individuals who stated that they had not felt such a need (question 4). Men who shaved every other day, and women who fixed their hair every other day, accepted treatment more often than did people who followed such grooming practices either less frequently or more frequently (questions 10a, 10b). Individuals who stated that they would be willing to accept treatment at the demonstration dental clinic accepted the treatment more frequently after it was offered than individuals who stated in advance that they would not be willing to accept such treatment (question 14).

The questions concerned with the psychology of older persons (questions 12 and 13) did not differentiate between individuals who accepted and those who did not accept treatment. The question related to the intensity of pain in dental care (question 8) also failed to discriminate between individuals who did and did not accept dental care.

\footnotetext{
"The first 80 of the total 310 patients interviewed were asked questions from a form which subsequently was revaluated and then revised. The answers from these individuals, therefore, could not be combined with the answers from the other 230 patients. Because of this procedure, the findings of relationship between answers to questions and acceptance of treatment were based on 230 persons in nursing homes. It should be noted that the number of persons who answered any one question, however, often was less than the total of 230. The total number in the comparisons, hence, will be fewer than 230 .
} 


\section{Relationship Between Acceptance of Dental Treatment and the Objective Dental Needs*}

A greater percentage of individuals accepted than rejected treatment both among individuals who had the greatest objective dental needs and those who had the lowest degree of objective dental needs (Figure 1). This situation was reversed among individuals who were intermediate in dental needs. A greater percentage of individuals from this intermediate group refused treatment than accepted treatment. These findings might be explained by assuming that individuals with lower objective needs had been provided previous dental care habitually and would, therefore, accept treatment more readily than those patients with intermediate objective needs. That this assumption was not. warranted will be seen by the lack of relationship between the stated frequency of care prior to entering the institution (question I) and the degree of objective dental need. No relationships were demonstrated between any of the demographic characteristics, acceptance, and objective dental need.

Figure 1

PERCENTAGE OF INDIVIDUALS ACCEPTING TREATMENT COMPARED WITH PERCENTAGE OF INDIVIDUALS REFUSING TREATMENT, BY DEGREE OF OBJECTIVE DENTAL NEED

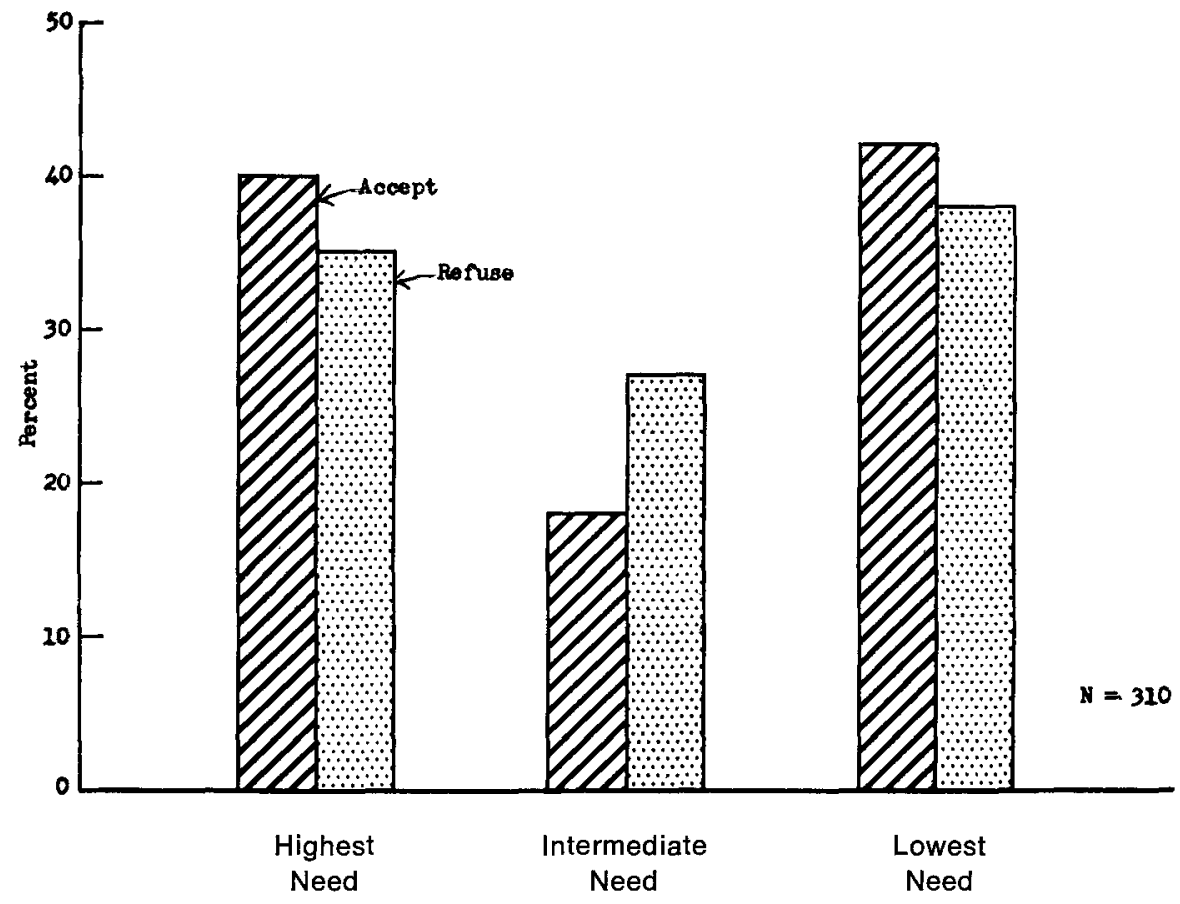

OBJECTIVE NEEDS

*A simple three-point scale was used for the definition of objective need. The clinical dental findings were stated in the larger report of the Kansas City Study. 


\section{Discussion}

The findings from this study will be found discussed under the headings of (1) the use of the interview as a predictive instrument, and (2) the use of the interview in suggesting hypotheses for further study.

\section{A Predictive Instrument}

The public health administrator needs to know which data will help him to predict the acceptance or refusal of treatment by which individuals or groups. This prediction has become relevant now that health administrators throughout the country are attempting to prepare for the additional load of patients expected as a result of Medicare. For maximum efficiency, the public health administrator also needs to know which data form the shortest, yet most reliable index for predicting the particular individual or group that will accept or refuse treatment. The next step, then, is to look for such combinations of findings in the data.

A single question discriminated most clearly individuals who accepted treatment from those who did not. This question determined the intention to accept treatment if offered (No. 14). Twenty-six individuals, who stated that they would accept treatment, ultimately refused treatment when it was offered; five individuals, who said initially that they would refuse treatment if it were offered, ultimately accepted treatment (Table 3).

TABLE 3

RELATIONSHIP BETWEEN STATEMENT OF WILLINGNESS

TO ACCEPT TREATMENT IF OFFERED, AND ACCEPTANCE OF TREATMENT

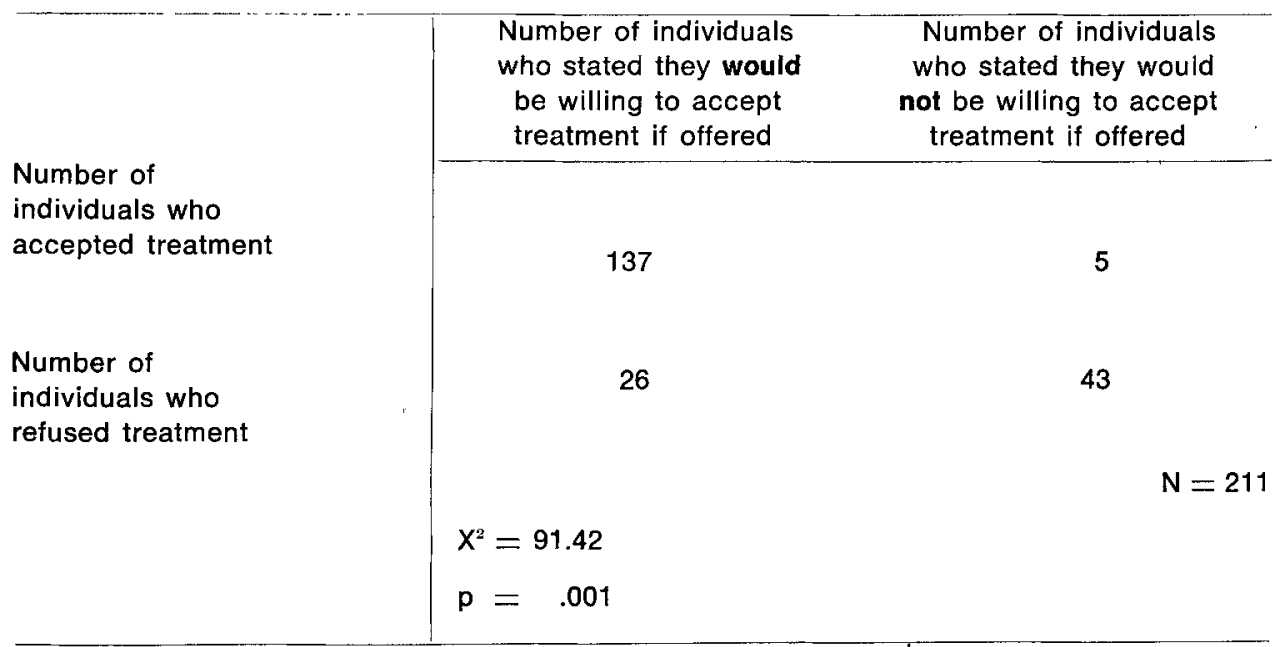

Twenty-three of the 26 individuals who stated that they would accept treatment and later refused treatment were females. This finding disclosed a significantly greater proportion of females to males than the proportion of females to males who accepted treatment after a statement of intention to do so. 
Sixteen of the 26 individuals who changed their minds stated that they practiced frequent grooming (shaving or fixing hair at least every other day) a finding which was significantly different from the proportion of such individuals who followed their stated intention (No. 11).

Though early expectations of a direct relationship between degree of objective dental need and acceptance of dental treatment were not found, one might still expect an inverse relationship between dental need and changing one's mind about going for dental care. Individuals with greater objective dental needs, it would seem, should be more likely to agree to get dental care, and then proceed to get it, than individuals with lesser objective dental needs. It will be seen in Figure 2 that the findings were not that simple. Among the individuals who stated that they intended to get dental care, the group with the most severe objective dental needs also included the greatest proportion of acceptors of treatment. The group with lowest needs, however, accepted treatment almost to the same extent as those with much greater objective dental needs. The group experiencing middle need was lowest in acceptance of treatment after expressing intention to get treatment.

Figure 2

\section{PERCENT ACCEPTING AND PERCENT REFUSING TREATMENT OF TOTAL GROUP WHO EXPRESSED INITIAL INTENTION TO ACCEPT TREATMENT, BY DEGREE OF OBJECTIVE DENTAL NEED}

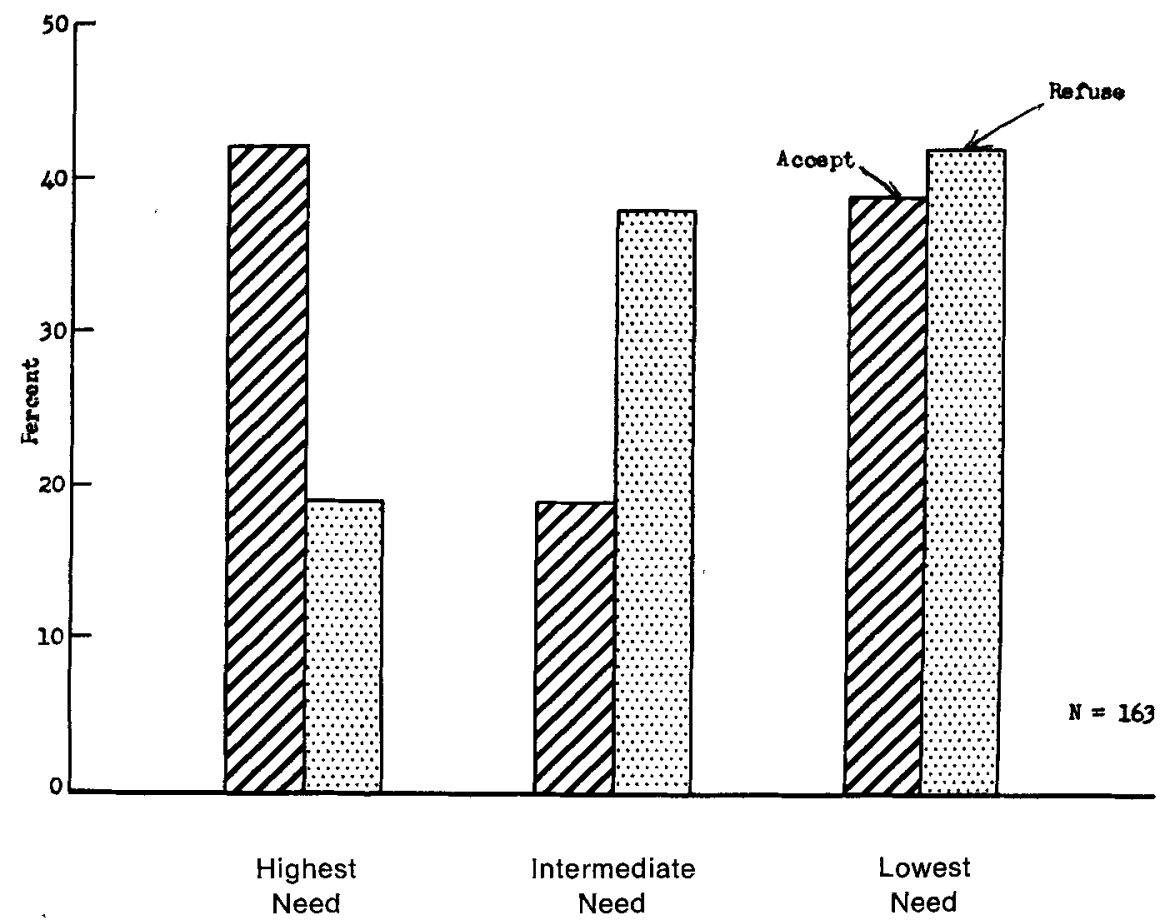


Restricting the analysis to individuals who agreed to get treatment, and then did not come for treatment, on the other hand, determined that the greater the objective need, the less often was the decision made not to come for treatment.

The number of persons who stated that they would refuse treatment, but when given the opportunity, actually accepted treatment, was very small (five in number). No comparisons could be made with individuals, hence, who stated that they would refuse treatment and actually did refuse it.

Summarizing this section, if a questionnaire of this type were to be found useful in other situations, the administrator would be most successful by directing his program to males who state: (1) they felt that they needed dental care; (2) frequently followed personal grooming-habits; and (3) would accept treatment if offered to them. A program of this nature, however, might be far too limited to suit the purposes of the administrator.

If he were to wish to increase the utilization of services, he would be wisest to use all persons who stated that they would accept treatment if it were offered. In this event, however, he would have to keep in mind that the females in the study reported, did change their minds. It now seems obvious that he should not allow the objective dental needs of patients to serve as a temptation to organize a program of treatment that is based only on high dental needs. Almost as many individuals with relatively low objective dental needs accepted treatment as those with high objective needs.

\section{Development of Hypotheses}

A certain number of questions emerged from this study which seemed worthy of intensive investigation. These questions will be raised, and tentative hypotheses will be submitted as answers:

\section{a. Why was the rate of participation by this population so high?}

Unfortunately, there are very few data available which can be compared directly with the present findings. The rate of acceptance in this study, however, was greater than that found for participation in programs of mass-screening ${ }^{1,2}$ and for programs of mass inoculation. The discrepancy even was greater if one recalls that the population under consideration for this study was composed mainly of persons 65 years of age and over. Findings from the Baltimore Chronic Illness Study ${ }^{1}$ indicated that 49.5 percent of participation developed among individuals 65 and over. The present findings revealed almost 70 percent of acceptance. The current findings, hence, were much closer to those reported for children's acceptance of free dental care ${ }^{i, 8}$ (about 85 percent) in Woonsocket, Rhode Island, and Richmond, Indiana, and to the findings on acceptance of physical examinations ${ }^{9,10}$ (over 80 percent) from the studies of the National Health Survey. The studies in which acceptance was high seem to possess in common: (1) services with no cost to the recipient, (2) services which are made extremely convenient for the recipient, and (3) services with readily seen applicability. Controlled studies need to be completed to demarcate which of these three factors account for the greatest extent of participation, or whether all three need to be used together.

\footnotetext{
"It should be noted that the medical part of the poverty-program (TAP) in Detroit, as well as in most other such programs, attempted to decentralize clinics and to provide free services for its clientele.
} 
Acceptance of care by patients in nursing homes may have been seen as a welcome diversion to this relatively friendless, potentially bored group of individuals. This thinking guided the questions concerned with (1) desire to make new friends (question 12), and (2) finding things to do to keep busy (question 13). Neither of these questions, unfortunately, discriminated between individuals who accepted and those who refused treatment. It was impossible to determine whether the lack of discrimination could have been produced by (1) inadequate communication to the respondents of the intent of the questions, (2) failure to interpret the opportunity to obtain dental care away from the nursing home as a way to occupy time or meet new people, or (3) the lack of relevance of the hypotheses which directed these questions. Research is needed on these factors.

\section{b. Why did males accept treatment more frequently than did females?}

The experience of dentists appears to indicate that females voluntarily seek dental care more frequently than do males. This experience was borne out partially by the data reported from the National Health Survey on the intervals elapsing since the last dental visit ${ }^{11}$ (Table 2, page 12). Cumulating the totals on dental practices presented in the publication of this survey it was found that more females ( 15 and over) had visited the dentist during the previous year at every age, except one, than had males at the same ages. The one exception, quite relevant for this study, was the age-grouping of 65 and over. Among persons 65 and over, a higher percentage of males had visited dentists within the last year than had females. The currently achieved data showed the same reversal as the one found in the data of the National Health Survey. More adult females have secured inoculations against polio than have adult males at every age. ${ }^{6}$

\section{c. Why did more Negroes accept treatment than did whites?}

In the studies reviewed by Rosenstock, et al., ${ }^{12}$ all but one showed higher inoculations against polio by whites than Negroes. The one study by Belcher ${ }^{13}$ found significantly higher participation in inoculations against polio by Negroes. It was explained as the result of great pressure exerted on the Negroes in the southern community studied. The Baltimore Chronic Illness findings detected a greater percentage of participation by nonwhites than by whites, but no explanation was postulated for this result. Research appears needed to ascertain the factors which explain this reversal. It appears sensible to speculate on the present tentative finding by conceiving of Negroes of the age studied as likely to act on any request as if it were a demand, because of their past experience with demands. There may have been more actual pressure exerted by operators of nursing homes on Negroes, than on whites, to procure the service. Negro operators of nursing homes might have been motivated by concern that their patients take advantage of an opportunity. If either perceived pressure or actual pressure did exist, the reasons for the finding might have been comparable to those reported by Belcher. ${ }^{13}$

It is possible that Negroes have become better adapted to health services under clinical auspices than have whites, since clinical services have been used largely by Negroes in the past. Such statements, especially since they are based on relatively few instances, are at best only speculation and need substantial assessment. It is hoped that such assessment will be made within- 
those poverty-programs in which the clientele is largely Negro. No data have been tound which answer such questions currently. Studies conducted by Cornely and Bigman ${ }^{1 \pm}$ and by Polgar and Cowles ${ }^{15}$ indicate that a large part of the Negro population typically fails to take advantage of low-cost facilities for health.

\section{d. Why did certain questions discriminate between individuals who accepted and those who refused treatment?}

The question, as stated, which discriminated most clearly between acceptors and nonacceptors of treatment, concerned the intention to accept treatment if offered (question 14). As was noted earlier, a single question, which discriminates so clearly, is a potentially useful aid to the administrator. From the point of view of research, however, the question which seems pertinent concerns the obviousness of such findings. This phenomenon did occur in the study carried out by the National Health Survey in the area of Washington, D C." In that survey, a very large number of individuals who agreed to make appointments for medical examinations kept their appointments.

Prior to accepting the assumption that such a question invariably predicts response, findings from other studies need to be reported as precautions. In a study carried out by National Analysts ${ }^{16 i}$ for Princeton University, the question about intended size of family proved to be a very poor predictor of the actual eventual size of the family. Questions of intention are no longer accepted as useful indicators of actual purchase by market-researchers. ${ }^{16}{ }^{16}$ Brown $^{17}$ has reported that only 52.4 percent of the intentions of housewives to purchase actually were carried out in a series of studies. Data from the series, conducted by the University of Michigan's Survey Research Center, which were concerned with the relationships between buyers' intentions and actual purchases, showed that one could predict the purchase of cars more correctly than other purchases, following the stated intentions, but even the intentions to purchase cars were not very useful as predictors. ${ }^{18,19,20}$ In a study conducted by the National Office of Vital Statistics, it was found that a large proportion of persons who reported an intention to obtain vaccination against polio within a two-month period did not, in fact, obtain it within that period.

Rather than accepting the findings reported as obvious, an investigator is faced instead with the question of why a statement of intention did discriminate so well in the current study. One needs to ask under what conditions and in which situations do questions of intention really predict behavior, and under what conditions and in which situations do they fail to predict behavior.

Two other questions produced different responses by acceptors and nonacceptors. It was noted that (1) acceptors had stated that they felt a need for care during their time in the institution more frequently than did the individuals who did not accept treatment (question 4) and that (2) acceptors practiced personal grooming at different rates than nonacceptors (questions 10a, 10b). Considering that there were no differences between acceptors and nonacceptors in regard to the amount of dental care received while institutionalized (question 2 ), or in objective dental need, a tentative explanation can be stated.

A theory of health-behavior, first stated by Hochbaum, ${ }^{21}$ and then reinterpreted by Kegeles ${ }^{2: 23}$ for dental behavior, might help explain these findings. It has been stated that an individual, in order to accept a health-activity, must be ready to act. This readiness for action is composed of (1) a feeling of suscepti- 
bility to the health problem, (2) a feeling that the health problem will be serious to the individual if he is afflicted, and (3) a feeling that actions are available to him to alleviate the seriousness of the problem which will benefit him by having taken these actions. It also was stated that some force, either from inside the individual, or from the outside world, must "cue" the individual before the readiness to act will be translated into action.

If the opportunity to procure dental care is seen as the necessary cue for action, it is conceivable that individuals in a population who perceived dental problems as serious (who felt that they needed dental care, even if objectively they did not need any more than individuals who did not feel the subjective need), or who saw dental problems as interfering with their appearance (and were quite concerned about their appearance as was noted by their efforts in personal grooming), would be more likely to accept such treatment. It would seem also that these individuals, in order to state that they would accept treatment if it were offered, also must have felt that dentists would be beneficial in relieving serious problems.

Because the theoretical model presented seems to fit the findings, it does not in any way preclude a more simple explanation of the findings through other kinds of theoretical statements. More research clearly is necessitated before the findings can be considered as more than simple empirical results.

\section{A Summary}

As part of a community's program of research and demonstration, a social survey was developed for predicting the acceptance of dental care by residents of nursing homes and by chronically ill, home-bound patients. This report includes findings from a study of 310 residents from 29 nursing homes. These individuals were examined for dental needs, interviewed by two trained interviewers, had dental services explained to them by a member of the staff, and finally were offered the services through the project's dental clinic.

It was found that, (1) 70 percent of the population accepted treatment, (2) Negroes accepted treatment more frequently than did whites, (3) persons under 75 accepted treatment more frequently than did persons over 75 , (4) males accepted treatment more frequently than did females, and (5) females under 80 accepted treatment more frequently than did females over 80 .

Responses to questions also showed that persons who eventually accepted treatment differed significantly from persons who eventually refused treatment. Their responses to questions showed that (1) more felt that they had needed dental care during their time in the institution, (2) more practiced frequent personal grooming, and (3) more stated that they would accept treatment if it were offered. No relation was found to exist between objective dental needs and acceptance of treatment.

The data then were combined in order to derive the most effective predictive device for the use of an administrator. It was found that males under 75 who stated that they would accept treatment if it were offered to them constituted the group that most readily accepted treatment. Negro males, though extremely small in number, accepted treatment significantly more frequently than white males. It was pointed out further that the best single prediction for acceptance of treatment was a statement of intention to accept treatment if it were offered. It was noted that there was a significant tendency for women to 
change their minds after stating that they would accept treatment if it were offered.

Finally, a series of questions was raised and some tentative hypotheses offered as starting points for additional research. In raising such questions, comparison was made with studies in which data on acceptance of care had been procured. A tentative conclusion was stated that studies, showing high participation, found in common: (1) care made extremely convenient to the respondent, (2) care which required no cost for the respondent, and (3) care which could be seen as readily applicable. A series of studies also was discussed in which questions of intention did and did not predict behavior. Finally, a theoretical formulation, developed from other studies, was utilized to point to some tentative reasons for respondents answering the questions as they had.

This extremely small sample from a relatively restricted population, unfortunately, reduced greatly the extent to which these findings could be generalized. An attempt to devise a relatively small number of questions which would be useful in predicting the extent to which older populations will seek healthcare seems imperative, now, in light of Medicare which shortly will be available.

\section{Bibliography}

1. Commission on Chronic Illness. Chronic illness in a large city. (In chronic illness in the United States. Cambridge, Mass., Harvard University Press, 1957. 4 vols., vol. 4. 620 p.)

2. Trussell, R. E., and Elinson, Jack. Chronic illness in a rural area. (In chronic illness in the United States. Cambridge, Mass., Harvard University Press, 1959. 4 vols., vol. 3. 440 p.)

3. Rubin, Theodore, Rosenbaum, Joseph, and Cobb, Sidney. The use of interview data for the detection of associations in field studies. J. Chronic Dis., 4:253-66, Sept. 1956.

4. Vermillion, J. R., Gailbreath, Mary N., and Rollins, P. L. Dental care for the chronically ill and aged; a community experiment. P.H.S. Publication, No. 899, Washington, Government Printing Office, 1962. $54 \mathrm{p}$.

5. Mainland, Donald, and Murray, I. M. Tables for use in fourfold contingency tests. Science, 116:591-4, Nov. 28, 1952.

6. Sirken, M. G., and Brenner, Berthold. Population characteristics and participation in the poliomvelitis vaccination program. Pub. Health Monogr., 61: I-37, 1960. (Also P.H.S. Publication No. 723, Washington, Government Printing Office, 1960.)

7. Law, F. E., Johnson, C. E., and Knutson, J. W. Studies on dental care services for school children - first and second treatment series. Woonsocket, R. I. Pub. Health Rep., 68:1192-8, Dec. 1953.

8. Waterman, G. E., and Knutson, J. W. Studies on dental care services for school children - third and fourth treatment series, Richmond, Ind. Pub. Health Rep., 69:247-54, Mar. 1954.

9. Borsky, P. M., and Sagen, O. K. Motivation toward health examination. Am. J. Pub. Health, 49:514-27, Apr. 1959.

10. U. S. National Health Survey. Attitudes toward cooperation in a health examination survey. Health Statistics, Series D6. P.H.S. Publication, No. 584-D6, Washington, Government Printing Office, 1961. $45 \mathrm{p}$.

11. . Dental care interval and frequency of visits, United States, July 1957 June 1959. Health Statistics. Series B14. Washington, Government Printing Office, 1960. $42 \mathrm{p}$.

12. Rosenstock, I. M., Derryberry, Mavhew, and Carriger. Barbara K. Why people fail to seek poliomyelitis vaccination. Pub. Health Rep., 74:98-103, Feb. 1959.

13. Belcher, J. C. Acceptance of the Salk polio vaccine. Rural Soc., 23:158-70, June 1958.

14. Cornely, P. B., and Bigman, S. K. Cultural considerations in changing health attitudes. D. C. Med. Ann., 30:19I-9, Apr. 1961.

15. Cowles, Wvlda, and Polgar, Steven. Health and communication in a Negro census tract. Social Problems, 10:228-36, Winter 1963.

16. National Analysts, Inc. Personal communication, Dr. Aaron J. Spector, 1960.

17. Brown, G. H. Measuring consumer attitudes towards products. p. 268-77. (In Ferber, Robert. and Wales, H. G., eds. Motivation and market behavior. Homewood, Ill., Irwin, 1958.437 p.) 
18. Kosobud, R, F., and Morgan, J. N., eds. Consumer behavior of individual families over two and three years. Ann Arbor, University of Michigan, Institute for Social Research, 1964. $208 \mathrm{p}$.

19. Lan'ing, J. B., and Withey, S. H. Consumer anticipations: their use in forecasting consumer behavior. p. 381-441. (In Conference on Research in Income and Wealth. Studies in income and wealth: short-term economic forecasting. National Bureau of Economic Research, Princeton University Press, 1955. 29 vols., vol. 17. 508 p.)

20. Leininger, C. A., Mueller, Eva L., and Wyss, H. Some uses of panel studies in forecasting the automobile market. Am. Statistical A. Proc., Business and Statistics Section, 117:409-21, 1957.

21. Hochbaum, G. M. Public participation in medical screening programs. A sociopsychological study. P.H.S. Publication, No. 572, Washington, Government Printing Office, 1958.: $23 \mathrm{p}$.

22. Kegeles, Stephen. Some motives for seeking preventive dental care. Am. Dent. A. J., 67:90-8, July 1963.

23. Why people seek dental care: a test of a conceptual formulation. J. Health and Human Behavior, 4:166-73, Fall 1963.

At the time this study was completed, Dr. Kegeles was chief of the Psychological Studies Section, Dr. Lotzkar served as the Clinical Dental Director of the project carried out in Kansas City, and Mr. Andrews served as the Director of the Kansas City Dental Project of the U. S. Public Health Service.

\title{
THE DENTAL PUBLIC HEALTH RESIDENCY*
}

\author{
By Robert L. Weiss, D.D.S., M.P.H.**
}

Training for students of dental public health, through an added year of supervised residency, indeed, has grown.

Last year at the Diplomates' Dinner-Meeting a report was presented of the developments during the first 16 months of experience with residencies. Tonight's report will be limited primarily to brief comments about the program of the past year, the current trends, developments and issues that are affecting the progress of the program, and the evaluatory process employed. Further details will be found in Appendixes $A$ and $B$.

\section{Residencies the Past Year}

This year 10 residents are in training in seven training-sites, the Dental Health Center and six state departments of health:

$\begin{array}{lll}\text { California } & \text { Georgia } & \text { New Jersey } \\ \text { Colorado } & \text { Kentucky } & \text { North Carolina }\end{array}$

Presented at a meeting of the Diplomates of the American Board of Dental Public Health, Las Vegas, November 6, 1965.

* Chief, Training Branch, Dental Health Center, San Francisco. 